\title{
The brave new world of eclipsing binary modeling
}

\author{
A. Prša \\ Villanova University, Dept. of Astrophysics and Planetary Science, 800 \\ Lancaster Ave, Villanova PA 19085
}

Received: October 31, 2019; Accepted: December 18, 2019

\begin{abstract}
This contribution highlights some of the open questions identified by the binary star community in attendance, based on a 45-min discussion in lieu of an ex-cathedra talk. As photometric and spectroscopic data accuracy reach unprecedented levels, it is crucial to identify and address these open questions. In what follows are concise minutes from the discussion with minimal commentary, in an effort to stimulate further discussion and promote focused studies dedicating to answering these open questions.
\end{abstract}

Key words: binaries: eclipsing - stars: fundamental parameters

\section{Introduction}

The landscape of eclipsing binary modeling is changing rapidly. Gone are the days where we would spend a few nights at a telescope, acquire an eclipsing binary light curve (and, if we have access to precious spectrographs, radial velocity curve), throw it all into the Wilson \& Devinney code and publish the resulting parameters. We know now how to do better: how to study parameter correlations and degeneracies robustly, how to estimate heuristic (instead of formal) errors and how to combine diverse, possibly heteroscedastic datasets efficiently and, above all, statistically correctly. We also came to realize that modeling noise is crucial for proper estimation of parameter posteriors and that simplifying assumptions regarding noise/instrumental processes are rarely - if ever - adequate. The data have become ubiquitous and precise, and the literal firehose of data about to hit us from the upcoming missions such as LSST is simply astounding. On top of all that, there is a number of open questions when modeling eclipsing binary observables that we have yet to fully answer. I discuss some of these questions in what follows below.

Thinking about what the "brave" new world of eclipsing binaries boils down to, I would argue that we need to:

- figure out why we do what we do: what new insights will the scientific study provide beyond simply having the data that make that study possible;

- critically examine what we consider current best practices over and over again; 
- adopt proven best practices from other fields - we have a lot to learn from fields like exoplanets that witnessed early adoption of advanced astrostatistical methods;

- report null results in peer-reviewed literature - let the community know when something does not work; and

- embrace the fact that eclipsing binary modeling is both difficult and computationally expensive.

For the purpose of this discussion, I asked the audience the following questions:

How do we handle data noise efficiently? With noise all but gaussian, do we rely on overly-simplified assumptions, incorporate an explicit noise model, resort to gaussian processes, or do something else entirely?

How do we parametrize our models? The efficiency of finding the right solution depends strongly on the choice of parameters that appear in the model. Is our choice of parameters the best one we can make?

How do we robustly solve the inverse problem? Using differential corrections and other deterministic minimizers benefits from speed, but suffers from the limited understanding of the parameter space topology; do we jump right into Bayesian inference, importance sampling, Markov Chain Monte Carlo sampling, nested sampling, or do the old methods still have clear merit?

What does it take to determine fundamental parameters to $1 \%$

$(\mathbf{0 . 1 \% ) ? ~ I f ~ d a t a ~ p r e c i s i o n ~ a l l o w s ~ t h i s ~ a c c u r a c y , ~ d o e s ~ t h e ~ m o d e l ~ a l l o w ~ i t ~ a s ~ w e l l ? ~}$ Is the precision in determined parameters really equivalent to their accuracy?

How do we handle a deluge of data coming our way? The ongoing and upcoming surveys such as Gaia, ZTF and LSST will swamp us with data. What are we hoping to learn from those data, and what needs to happen to enable us to process those data?

How do we classify binaries based on their light curve morphology? The crude separation of all binaries into detached, semi-detached and contact is barely more informative than claiming that the object is an eclipsing binary; how can we do better?

Do we handle contact binaries correctly? There is a large number of simplifying assumptions that are difficult to justify, most notably the "merging" in the neck area. Can we do better?

How do we bridge binarity with stellar populations and stellar

evolution? With only a few notable exceptions, stellar evolution focuses on single stars; what needs to happen to enable the reliable model of binary 
and multiple populations and their evolution, and how would such models be able to constrain eclipsing binary modeling further?

Forty-five minutes is obviously too short a time to answer all these questions, so we focused on a subset deemed most pertinent by the audience. The minutes of the discussion are provided below.

\section{Discussion notes}

- Why are we doing what we are doing? What are the fundamental questions we are trying to answer with binary star science?

- Pushing the accuracy of fundamental parameters $(3 \% \rightarrow 1 \% \rightarrow 0.1 \%)$

- The role of Helium - He fraction affects evolution, mass/luminosity and therefore limits accuracy. The tables of evolutionary models do not include variable He abundance. He abundance determined by theory but not from observations

- How do we define effective temperature from spectroscopy?

- Understanding the role of binary interactions - a lot if left unanswered because of the limitations on accuracy

- Stellar evolution in binary stars

- Extreme low-mass WD forming in 1 AU orbits where they should not test speculations of stable mass transfer that leaves them in those orbits

- Paying more attention to observations to get answers

- Problems that arise in modeling when introducing rotational models

- Magnetic fields

- Constraints from EBs also constrain single star parameters

- Handling data noise

- If the original model is slow, it gets harder to add more modeling on top of it $\rightarrow$ optimize the model (or buy a supercomputer)

- Include the noise model

- Our field is doing well compared to other fields - (ground-based) observations tend to not have any systematic error due to standard processing techniques. This does not apply to surveys that push the limit of accuracy

- We should not assume our data uncertainties are realistic 
- More advanced techniques (AO, speckle) require detailed modeling of the noise in concert with instrumentation (not simple shot noise!), therefore returning the uncertainty as one number is unreliable

- Is the burden on the observers or modelers to handle this?

- Modelers should understand exactly what is going on in the instrument (beyond empirical evidence) but it can get complicated

- Division of labor between observers and users - instrument scientists should deliver reduced data and noise models. How do we get there? Impose standards

- Use standards to retrieve the noise model from observations where we know it should not be

- Disentangling instrumental trends from actual astrophysical signals?

- Pipelines: users overlook the uncertainties that the pipeline introduces should also go in the noise model

- Large surveys: ZTF, Gaia, LSST - how do we handle data?

- Do we need millions EBs and what do we do with them?

- If we do not have enough follow-up with RVs it does not matter if we have millions of LCs

- Advantage: we will finally be able to see the stellar populations on the low-mass end

- Make reasonable approximations to get masses, radii, distances (better to have 10,000 objects with precision of $15 \%$ than 10 with $3 \%$ )

- Find the ones that are really special (example SDB with non-conservative mass transfer)

- Classical novae and follow-up triggers - not much incentive for observers to observe novar in quiescence and these surveys can fill those gaps. TESS is doing this right now, however the problem remains that people do not publish data of these objects in quiescence

- The choice of parameters

- We rely on parameters driven by physics, but with that impose correlations and degeneracy implicitly which limits our ability to derive accurate parameters

- Parameters with respect to each other: how do we address treating parameters/parameter ranges equally when they probably should not be? 
- Parameters with respect to the data: test whether data are sensitive to our parameters of choice before fitting for them

\section{Acknowledgements.}

The author expresses his utmost thanks to Dr. A. Kochoska for taking discussion notes, and to W. Van Hamme for endorsing the unconventional style used for this talk.

\section{References}

Wilson, R. E. \& Devinney, E. J., Realization of Accurate Close-Binary Light Curves: Application to MR Cygni. 1971, Astrophys. J., 166, 605, DOI: 10.1086/150986 